\title{
ORIGINAL
}

\section{EVOLUCIÓN DE LAS DIFERENCIAS SOCIOECONÓMICAS EN LA UTILIZACIÓN Y ACCESIBILIDAD DE LOS SERVICIOS SANITARIOS EN ESPAÑA ENTRE 1987 Y 1995/97}

\author{
Lourdes Lostao (1), Enrique Regidor (2), M. ${ }^{a}$ Elisa Calle (2), Pedro Navarro (2) y Vicente \\ Domínguez (2) \\ (1) Departamento de Sociología. Universidad Pública de Navarra. \\ (2) Departamento de Medicina Preventiva y Salud Pública. Facultad de Medicina. Universidad Complutense de Madrid.
}

\section{RESUMEN}

Fundamento: El objetivo del presente trabajo es evaluar la evolución de la utilización y accesibilidad de los servicios sanitarios en España, entre 1987 y 1995/1997, en grupos con diferentes características socioeconómicas.

Métodos: Los datos utilizados proceden de las Encuestas Nacionales de Salud realizadas por el Ministerio de Sanidad y Consumo a la población adulta en los años 1987, y 1995/1997. Se ha agregado la información de 1995 y 1997 debido al diferente tamaño muestral, de forma que las estimaciones así obtenidas son una media de ambas. La población analizada ha sido la de los individuos mayores de 24 años de edad. Se han estudiado la consulta médica, la hospitalización, la consulta al dentista y la consulta al ginecólogo, el tiempo invertido en llegar a la consulta, el tiempo de espera en la misma y el tiempo de espera para un ingreso hospitalario ordinario. Las características socioeconómicas utilizadas han sido el nivel de estudios y el grupo socioeconómico de los entrevistados. La medida de la asociación estimada entre la utilización de servicios y las características socioeconómicas fue la razón de porcentajes, mediante regresión binomial. Igualmente, se estimó el índice relativo de desigualdad como medida resumen de la desigualdad.

Resultados: La consulta médica fue más frecuente en los individuos sin estudios y en los grupos socioeconómicos bajos, en uno y en otro período, mientras que la consulta al dentista y la consulta al ginecólogo fueron más frecuentes en los individuos con estudios superiores y en los grupos socioeconómicos altos en ambos períodos. No se encontraron diferencias socioeconómicas estadísticamente significativas en la frecuencia de hospitalización en ambos períodos. Tanto en 1987 como en 1995/1997 no se hallaron diferencias estadísticamente significativas entre los distintos grupos socioeconómicos en el tiempo de llegada a la consulta $(p>0,05)$, pero sí en el tiempo de espera en la consulta $(\mathrm{p}<0,05)$. En el segundo período desaparecieron las diferencias socioeconómicas en el tiempo de espera para ingreso hospitalario ordinario que se observaron en el primer período.

Correspondencia:

Lourdes Lostao

Departamento de Sociología

Universidad Pública de Navarra

Campus de Arrosadía

Pamplona

31006 Navarra

Correo electrónico: 1lostao@unavarra.es
ABSTRACT

\section{Evolution of the socio-economic differences in the use and accessibility of health services in Spain between 1987 and 1995-97}

Background: The objective of this study is to evaluate the trend in the use and accessibility of health services in Spain between 1987 and 1995-1997 in groups with different socio-economic characteristics.

Methods: The data used are taken from National Health Surveys carried out on the adult population in 1987 and 1995-97 by the Ministry of Health and Consumer Affairs. The information for 1995 and 1997 has been combined due to the different sample size, in such a way that the estimates so obtained are the mean from both years. Population analysed has been people aged 24 years and older. The following have been studied: visits to the doctor, hospitalization, visits to the dentist and the gynecologist, the time taken to reach the visits to the doctor, and the waiting time in both the visits to the doctor and ordinary hospital admission. The socio-economic characteristics used are the level of education and the socio-economic group of the respondents. The measure of the association calculated between use of services and the socio-economic characteristics was the percentage ratio estimated by binomial regression. In addition, the relative index of inequality was estimated as a global measure of inequality.

Results: Visits to the doctor were the most frequent factor in people without academic qualifications and the lower socio-economic group in the two periods, while visits to the dentist and the gynecologist were more frequent in the higher socio-economic groups in both periods. Statistically significant differences in the frequency of hospitalization in the two periods were not found. Neither in 1987 nor $1995 / 1997$ were statistically significant differences found between the different socio-economic groups as regards the time taken to visits to the doctor $(p>0,05)$, although there were in waiting time in visits to the doctor $(p<0,05)$. In the second period the socio-economic differences observed in the first period in waiting time for ordinary hospital admission disappeared. 
Conclusiones: En la segunda mitad de los años noventa se observa el mismo perfil socioeconómico en la utilización de los servicios sanitarios y en los tiempos de espera para acceder a los mismos que en la segunda mitad de los años ochenta, con la excepción del tiempo de espera para ingreso hospitalario ordinario en el segundo período.

Palabras clave: Utilización de servicios. Accesibilidad a los servicios sanitarios. Evolución. Nivel de estudios. Grupos socioeconómicos.
Conclusions: In the second half of the 1990s the same socio-economic profile was observed in the use of health services and the time spent waiting to receive them, as in the second half of the 1980 s, with the exception of waiting time for ordinary hospital admission in the second period.

Key words: Use of services. Accessibility of health services. Evolution. Level of education. Socio-economic groups.

\section{INTRODUCCIÓN}

La utilización y la accesibilidad de los servicios sanitarios por parte de todos los grupos sociales de la población ha sido y es objeto de debate en muchos países industrializados ${ }^{1-5}$. En los países europeo-occidentales, caracterizados por Estados del Bienestar altamente desarrollados, en los que existe cobertura de la asistencia sanitaria pública, las instituciones públicas garantizan el derecho social «a la protección de la salud». En este sentido, investigaciones realizadas acerca de la accesibilidad y utilización del sistema sanitario reflejan que, aunque se ha logrado alcanzar una utilización similar de los servicios de atención primaria y hospitalaria por parte de distintos grupos socioeconómicos, todavía persisten diferencias en el uso de los servicios preventi$\operatorname{vos}^{6-11}$.

La mayor parte de esos estudios se refieren a la situación en los años ochenta o principios de los noventa pero, recientemente, también se han empezado a publicar algunos estudios que comparan las diferencias socioeconómicas en la utilización de los servicios de atención primaria en la década de los ochenta con la década de los noventa ${ }^{12-15}$. Así, en Gran Bretaña y en Francia ${ }^{12-13}$, se ha puesto de manifiesto que en los primeros años de los noventa continuaba el mismo patrón observado diez años antes, esto es, ausencia de diferencias socioeconómicas en la utilización de estos servicios; en cambio, en Suecia, se encontró que los grupos socioeconómicos altos en los años noventa tenían una tasa de utilización de servicios de medicina general más alta que los grupos socioeconómicos bajos a igual grado de necesidad, a pesar de que en los años ochenta no se encontraron diferencias en la utilización de tales servicios. Se ha sugerido que los principales cambios en el mercado laboral y las políticas de salud realizadas podrían explicar las tendencias observadas en la utilización de servicios en Suecia ${ }^{13}$, así como los distintos intentos llevados a cabo en este país para aumentar el numero de médicos en atención primaria ${ }^{14-15}$.

En España, algunos estudios a nivel municipal con información de finales de los ochenta y principios de los noventa mostraron la existencia de diferencias socioeconómicas en la consulta médica y en la hospitalización ${ }^{16-17}$. Estudios a nivel estatal no encontraron diferencias socioeconómicas en la consulta médica y en la hospitalización, pero sí en el uso de servicios preventivos y en el uso de servicios médicos priva$\operatorname{dos}^{11,18-20}$. Igualmente se encontraron diferencias socioeconómicas en el tiempo de espera para la consulta médica y en el tiempo de espera para ingreso hospitalario ${ }^{11}$. Dado que a lo largo de los años noventa han tenido lugar diferentes tipos de intervenciones en el Sistema Nacional de Salud (planes de actuación para disminuir las listas de espera para ingreso hospitalario, cita previa para disminuir el tiempo de espera para una consulta, programas de detección precoz del cáncer de mama, etcétera), sería de gran valor conocer la evolución de las diferencias socioeconómicas en la utilización y accesibilidad de los servicios sanitarios, ya que la aplicación de determinadas políticas sanitarias podría redundar en un aumento de las desigualdades en la utilización de los servi- 
cios. De acuerdo a este interés, el objetivo del presente trabajo sería evaluar la evolución de las diferencias en el uso y accesibilidad de los servicios sanitarios en España, entre 1987 y 1995/1997, en grupos con diferentes características socioeconómicas.

\section{MATERIAL Y MÉTODOS}

\section{Fuentes de datos}

Los datos proceden de las encuestas nacionales de salud realizadas por el Ministerio de Sanidad y Consumo a la población adulta en los años 1987, 1993, 1995 y 1997. En las encuestas nacionales de salud de 1987 y 1993 se entrevistó una muestra domiciliaria de 30.000 y 21.120 personas, respectivamente, representativas de la población española no institucionalizada de 16 y más años, mientras que en las encuestas de 1995 y 1997 el tamaño muestral fue de 6.400 personas, respectivamente. En 1987 y 1993 las muestras estuvieron formadas por 50 submuestras provinciales. En cada una de esas submuestras el procedimiento muestral fue polietápico y estratificado por tamaño de hábitat, con selección de las unidades primarias de muestreo (municipios) y de las unidades secundarias (secciones censales) de forma aleatoria proporcional. Finalmente, las últimas unidades muestrales (individuos) se eligieron por cuotas de edad y sexo. Debido a que la fracción de muestreo no fue idéntica en cada provincia, a cada individuo de la muestra se le asignó un coeficiente de ponderación en función de la provincia de residencia. En cambio en 1995 y en 1997 la muestra fue autoponderada, ya que tanto en uno como en otro año se seleccionó una sola muestra a nivel nacional, utilizando el mismo procedimiento de selección que las encuestas de 1987 y 1993 . Un 10\% de las entrevistas en 1987 y 1993 y un $15 \%$ de las entrevistas en 1995 y en 1997 se realizaron después de que uno o más de los individuos seleccionados rehusaran contestar al cuestionario.
El presente estudio se ha restringido a la población mayor de 24 años debido a que una de las variables elegidas para el análisis es el nivel de estudios terminado y antes de esa edad la mayoría de los individuos que cursan estudios de tercer grado no los han finalizado.

\section{Variables de estudio}

En este trabajo se han investigado la consulta médica, la hospitalización, la consulta al dentista y la consulta al ginecólogo, el tiempo invertido en llegar a la consulta del médico, el tiempo de espera en la misma y el tiempo de espera para ingreso hospitalario ordinario. Las encuestas nacionales de salud recogen información de esas características mediante las siguientes preguntas: ¿ha consultado con algún médico por algún problema, molestia o enfermedad suya en las dos últimas semanas?; en la última consulta realizada ¿cuánto tiempo tardó, aproximadamente, en llegar desde su domicilio al lugar de la consulta? y ¿cuánto tiempo tuvo que esperar en el lugar de la consulta desde que llegó hasta que fue atendido por el médico?; ¿ha estado hospitalizado como paciente, al menos durante una noche, en los últimos doce meses? En cuanto al tiempo de espera para ingreso hospitalario, a aquellos que señalaban el último ingreso como ordinario, programado o por lista de espera, en la encuesta de 1987 se preguntaba ¿cuántos días, aproximadamente, transcurrieron desde el día de la consulta en la que el médico le indicó que ingresara en el hospital hasta el día del ingreso?; en cambio, en las encuestas de 1995 y 1997, a aquellos que señalaban que en el último ingreso hospitalario estaban en la lista de espera, se les preguntaba ¿cuánto tiempo en meses había estado en la lista de espera? La información sobre la consulta al dentista se obtuvo mediante la pregunta: ¿ha consultado al dentista, protésico o higienista dental para examen, consejo o tratamiento de problemas de su dentadura en los últimos tres meses? La consulta al ginecólogo no se in- 
vestigó en la encuesta de 1987; por ello, con el objeto de evaluar la tendencia de esta característica, se utilizaron los datos que proporciona la encuesta nacional de salud de 1993. En las encuestas, la información referente a la consulta al ginecólogo se obtuvo sólo de las mujeres con la siguiente pregunta ¿ha acudido alguna vez al ginecólogo? y a las que contestaron afirmativamente, se les preguntó cuándo consultaron por última vez por un motivo diferente al embarazo o al parto. En el presente estudio, sólo se investigó la consulta realizada al ginecólogo en el último año.

Las características socioeconómicas que se han analizado han sido el nivel de estudios y el grupo socioeconómico. El nivel de estudios se obtuvo de manera diferente en la encuesta de 1987 y en las encuestas de 1995 y 1997, por ello, para poder comparar los dos períodos, los individuos se han agrupado en tres categorías: sin estudios/estudios de primer grado, estudios de segundo grado y estudios de tercer grado. Por su parte, para agrupar a los individuos según el grupo socioeconómico se ha utilizado una clasificación que tiene en cuenta la ocupación y la situación laboral del cabeza de familia (Carmen Rodríguez, Propuesta de clasificación de nivel económico, no publicada). Así, cada entrevistado fue asignado a uno de los siguientes cinco grupos en los que se clasificó al cabeza de familia: bajo (V): estudiantes, sus labores, parados que buscan su primer empleo y trabajadores manuales no cualificados parados o jubilados; medio-bajo (IV): trabajadores manuales no cualificados ocupados y trabajadores manuales cualificados parados o jubilados; medio (III): trabajadores manuales cualificados ocupados y mandos intermedios, empleados administrativos y trabajadores autónomos parados; medio-alto (II): mandos intermedios, empleados administrativos y trabajadores autónomos ocupados y directivos, empresarios y profesionales parados o jubilados; y alto (I): directivos, empresarios y profesionales ocupados.

\section{Análisis estadístico}

Con el fin de compensar las diferencias en el tamaño muestral, se agregaron las encuestas de 1995 y 1997, de tal forma que las estimaciones del primer período se han obtenido con los datos de la encuesta de 1987, excepto en el caso de la consulta al ginecólogo que se utilizó la encuesta de 1993, mientras que las estimaciones del segundo período se basan en los datos procedentes de la unión de las encuestas de 1995 y 1997. La unión de los individuos seleccionados en 1995 y 1997 con muestreo similar permite aumentar el número de efectivos en los análisis, aunque es preciso tener en cuenta que las estimaciones obtenidas son una media de las dos encuestas.

Se calculó el porcentaje de individuos que utilizó cada servicio sanitario en cada grupo socioeconómico y en cada período así como la asociación entre el grupo socioeconómico y la utilización de cada servicio ajustada por edad, sexo y una medida de necesidad. En la consulta médica y en la hospitalización la medida de necesidad fue la presencia de enfermedad crónica, mientras que en la consulta al dentista la medida de necesidad fue el estado de la dentadura. Por su parte, en la consulta al ginecólogo no se encontró ninguna variable indicadora de la necesidad de este tipo de asistencia en el cuestionario. No se incluyó como medida de necesidad la autovaloración del estado de salud, ya que esta variable tiende a reflejar el bienestar general y no únicamente el estado de salud. Por otro lado, se incluyó el tipo de cobertura sanitaria (publica y doble — privada y pública-), pero los modelos estadísticos utilizados no convergieron, con lo que finalmente fue excluida del análisis. Igualmente, se planteó la posibilidad de realizar el análisis por separado en hombres y en mujeres, sin embargo debido a que el patrón de utilización de los servicios sanitarios según la clase social en hombres y en mujeres es muy similar ${ }^{19}$, se decidió analizarlos de forma conjunta.

La medida de asociación estimada fue la razón de porcentajes calculada mediante re- 
gresión binomial y tanto en uno como en otro período se obtuvo la significación estadística de su tendencia. Además, para comparar los resultados encontrados en cada período se ha utilizado una medida resumen de las desigualdades, denominada índice relativo de desigualdad, en la que además de la medida de efecto se tiene en cuenta, el tamaño de cada uno de los grupos socioeconómi$\cos$.

Posteriormente se estimaron la media y la mediana de cada uno de los tiempos de espera. Debido a que el número de efectivos es menor, ya que sólo se tiene en cuenta a los que han utilizado los servicios sanitarios, el nivel de estudios se agregó en dos categorías y el grupo socioeconómico se agrupó en tres categorías con el objeto de reducir el margen de incertidumbre en las estimaciones. Para comparar las diferencias socioeconómicas en los tiempos de espera en 1987 y en 1995/97 se empleó un modelo de regresión multiple para cada tiempo de espera y período, en el que la variable dependiente fue el logaritmo del tiempo y como variables independientes se incluyeron la edad, el sexo y la variable socioeconómica (nivel de estudios o grupo socioeconómico). El nivel de estudios y el grupo socioeconómico se introdujeron como variables ordinales, de tal forma que la exponenciación de su coeficiente mediante la formula $(\exp -1) * 100$ produce la diferencia porcentual en los tiempos de espera asociada al nivel de estudios o al grupo socioeconómico. En los coeficientes de regresión , los valores de p superiores a 0,05 se consideraron no estadísticamente significativos.

Todos los cálculos estadísticos fueron realizados con $\mathrm{SAS}^{21}$. Todas las estimaciones correspondientes a los años 1987 y 1993 fueron ponderadas por el coeficiente de ponderación provincial. En las estimaciones correspondientes a 1995/97 no fue necesario ya que como se ha señalado las muestras fueron autoponderadas a nivel estatal.

\section{RESULTADOS}

En la tabla 1 aparece la distribución de los individuos según el nivel de estudios y el grupo socioeconómico en uno y otro período. Se observa que, con respecto a 1987 , en 1995/97 ha aumentado el porcentaje de individuos con estudios de segundo y tercer grado y ha disminuido el porcentaje de individuos sin estudios o con estudios de primer grado. Igualmente ha aumentado el porcen-

Tabla 1

Número de individuos analizados y distribución porcentual de los mismos según el nivel de estudios y el grupo socioeconómico. Población española mayor de 24 años, 1987 y 1995/97

\begin{tabular}{|c|c|c|c|c|}
\hline & \multicolumn{2}{|c|}{1987} & \multicolumn{2}{|c|}{ 1995/97 } \\
\hline & Número & $\%$ & Número & $\%$ \\
\hline \multicolumn{5}{|l|}{ Nivel de estudios } \\
\hline Sin estudios/Primer grado & 17.963 & 76,6 & 5.642 & 63,4 \\
\hline Segundo grado & 3.344 & 14,3 & 2.097 & 23,6 \\
\hline Tercer grado & 2.139 & 9,1 & 1.159 & 13,0 \\
\hline \multicolumn{5}{|l|}{ Grupo socioeconómico } \\
\hline V. Bajo & 3.740 & 17,1 & 1.505 & 14,9 \\
\hline IV. Medio-Bajo & 5.089 & 23,3 & 2.110 & 20,9 \\
\hline III. Medio & 6.549 & 30,0 & 2.926 & 29,0 \\
\hline II. Medio-Alto & 5.496 & 25,2 & 2.409 & 23,8 \\
\hline I. Alto & 960 & 4,4 & 1.152 & 11,4 \\
\hline
\end{tabular}


taje de individuos pertenecientes al grupo socioeconómico alto y ha disminuido el porcentaje de individuos pertenecientes a los grupos socioeconómicos medio-bajo y bajo.

En las tablas 2 y 3 puede observarse el tanto por ciento de individuos que utilizó cada uno de los servicios sanitarios según el nivel de estudios y el grupo socioeconómico, así como la razón de porcentajes que evalúa la asociación entre el uso de estos servicios y cada variable socioeconómica. La frecuencia de consulta médica y la magnitud de la razón de porcentajes aumenta conforme se desciende en la categoría socioeconómica en uno y otro período; en ambos períodos la tendencia de la razón de porcentajes fue estadísticamente significativa $(\mathrm{p}<0,05)$. El índice relativo de desigualdad en la consulta médica según el nivel de estu-

Tabla 2

Porcentaje de individuos que utilizó diferentes servicios sanitarios según el nivel de estudios y asociación (razón de porcentajes) entre la utilización de los servicios sanitarios y el nivel de estudios en 1987 y en 1995/97. Población mayor de 24 años

\begin{tabular}{|c|c|c|c|c|c|c|c|c|}
\hline & \multicolumn{4}{|c|}{1987} & \multicolumn{4}{|c|}{ 1995/97 } \\
\hline & $\%$ & $R P(1)$ & \multicolumn{2}{|c|}{ IC $95 \%$} & $\%$ & $R P(1)$ & \multicolumn{2}{|c|}{ IC $95 \%$} \\
\hline \multicolumn{5}{|l|}{ Consulta médica } & & & & \\
\hline Sin estudios/primer grado & 21,5 & 1,31 & $(1,18$ & $1,46)$ & 28,4 & 1,15 & $(1,01$ & $1,30)$ \\
\hline Segundo grado & 15,1 & 1,20 & $(1,06$ & $1,36)$ & 20,3 & 1,07 & $(0,92$ & $1,24)$ \\
\hline Tercer grado & 12,5 & 1,00 & & & 18,4 & 1,00 & & \\
\hline Test de tendencia (p) & & 0,0001 & & & & 0,0001 & & \\
\hline Índice relativo de desigualdad & & 1,40 & $(1,22$ & $1,62)$ & & 1,22 & $(1,03$ & $1,43)$ \\
\hline \multicolumn{9}{|l|}{ Hospitalización } \\
\hline Sin estudios/primer grado & 7,4 & 1,00 & $(0,86$ & $1,18)$ & 9,2 & 1,19 & $(0,95$ & $1,51)$ \\
\hline Segundo grado & 8,3 & 1,05 & $(0,89$ & $1,26)$ & 8,6 & 1,20 & $(0,94$ & $1,54)$ \\
\hline Tercer grado & 7,2 & 1,00 & & & 7,1 & 1,00 & & \\
\hline Test de tendencia (p) & & 0,8100 & & & & 0,2100 & & \\
\hline Índice relativo de desigualdad & & 0,97 & $(0,78$ & $1,21)$ & & 1,25 & $(0,93$ & $1,69)$ \\
\hline \multicolumn{9}{|l|}{ Consulta al dentista } \\
\hline Sin estudios/primer grado & 11,5 & 0,60 & $(0,54$ & $0,65)$ & 13,0 & 0,65 & $(0,57$ & $0,74)$ \\
\hline Segundo grado & 16,7 & 0,82 & $(0,73$ & $0,91)$ & 18,3 & 0,85 & $(0,74$ & $0,98)$ \\
\hline Tercer grado & 19,7 & 1,00 & & & 21,6 & 1,00 & & \\
\hline Test de tendencia (p) & & 0,0001 & & & & 0,0001 & & \\
\hline Índice relativo de desigualdad & & 0,45 & $(0,39$ & $0,52)$ & & 0,53 & $(0,44$ & $0,63)$ \\
\hline \multicolumn{9}{|l|}{ Consulta al ginecólogo (2) } \\
\hline Sin estudios/primer grado & 36,5 & 0,83 & $(0,76$ & $0,90)$ & 41,0 & 0,76 & $(0,69$ & $0,84)$ \\
\hline Segundo grado & 60,3 & 1,03 & $(0,95$ & $1,13)$ & 63,1 & 1,07 & $(0,98$ & $1,17)$ \\
\hline Tercer grado & 64,7 & 1,00 & & & 60,9 & 1,00 & & \\
\hline Test de tendencia (p) & & 0,0001 & & & & 0,0001 & & \\
\hline Índice relativo de desigualdad & & 0,70 & $(0,63$ & $0,78)$ & & 0,61 & $(0,53$ & $0,69)$ \\
\hline
\end{tabular}

(1) La razón de porcentajes está ajustada por edad, sexo y presencia de alguna enfermedad crónica en la consulta médica y en la hospitalización; por edad, sexo y estado de la dentadura en la consulta al dentista y por edad en la consulta al ginecólogo.

RP: razón de porcentajes; IC 95\%:intervalo de confianza al 95\%.

(2) En la consulta al ginecólogo los datos no corresponden a 1987 sino a 1993, debido a que en 1987 no se obtuvo este tipo de información en la Encuesta Nacional de Salud. 
Tabla 3

Porcentaje de individuos que utilizó diferentes servicios sanitarios según el grupo socioeconómico y asociación (razón de porcentajes) entre la utilización de los servicios sanitarios y el nivel de estudios en 1987 y en 1995/97. Población mayor de 24 años

\begin{tabular}{|c|c|c|c|c|c|c|c|c|}
\hline & \multicolumn{4}{|c|}{1987} & \multicolumn{4}{|c|}{ 1995/97 } \\
\hline & $\%$ & $R P(1)$ & $I C 9$ & $5 \%$ & $\%$ & $R P(1)$ & $I C S$ & $5 \%$ \\
\hline \multicolumn{5}{|l|}{ Consulta médica } & & & & \\
\hline V. Bajo & 27,2 & 1,59 & $(1,34$ & $1,89)$ & 33,0 & 1,17 & $(1,01$ & $1,34)$ \\
\hline IV. Medio-Bajo & 21,1 & 1,47 & $(1,24$ & $1,74)$ & 30,1 & 1,26 & $(1,10$ & $1,44)$ \\
\hline III. Medio & 18,0 & 1,31 & $(1,11$ & $1,55)$ & 25,8 & 1,15 & $(1,01$ & $1,32)$ \\
\hline II. Medio-Altos & 15,5 & 1,21 & $(1,02$ & $1,44)$ & 21,8 & 1,05 & $(0,91$ & $1,20)$ \\
\hline I. Alto & 12,0 & 1,00 & & & 19,3 & 1,00 & & \\
\hline Test de tendencia $(\mathrm{p})$ & & 0,0001 & & & & 0,0012 & & \\
\hline Índice relativo de desigualdad & & 1,44 & $(1,31$ & $1,58)$ & & 1,18 & $(1,05$ & $1,32)$ \\
\hline \multicolumn{9}{|l|}{ Hospitalización } \\
\hline V. Bajo & 7,6 & 0,89 & $(0,70$ & $1,13)$ & 10,9 & 1,17 & $(0,90$ & $1,53)$ \\
\hline IV. Medio-Bajo & 8,1 & 0,99 & $(0,79$ & $1,24)$ & 9,7 & 1,17 & $(0,91$ & $1,50)$ \\
\hline III. Medio & 7,7 & 0,93 & $(0,74$ & $1,16)$ & 9,0 & 1,17 & $(0,92$ & $1,49)$ \\
\hline II. Medio-Alto & 6,4 & 0,80 & $(0,63$ & $1,01)$ & 7,9 & 1,05 & $(0,82$ & $1,36)$ \\
\hline I. Alto & 7,9 & 1,00 & & & 7,1 & 1,00 & & \\
\hline Test de tendencia $(\mathrm{p})$ & & 0,2795 & & & & 0,155 & & \\
\hline Índice relativo de desigualdad & & 1,11 & $(0,94$ & $1,32)$ & & 1,12 & $(0,90$ & $1,40)$ \\
\hline \multicolumn{9}{|l|}{ Consulta al dentista } \\
\hline V. Bajo & 9,7 & 0,59 & $(0,50$ & $0,70)$ & 10,1 & 0,51 & $(0,42$ & $0,61)$ \\
\hline IV. Medio-Bajo & 11,2 & 0,61 & $(0,53$ & $0,71)$ & 13,3 & 0,63 & $(0,54$ & $0,74)$ \\
\hline III. Medio & 14,0 & 0,75 & $(0,65$ & $0,85)$ & 14,1 & 0,63 & $(0,55$ & $0,73)$ \\
\hline II. Medio-Alto & 14,7 & 0,76 & $(0,66$ & $0,87)$ & 17,0 & 0,76 & $(0,66$ & $0,87)$ \\
\hline I. Alto & 19,3 & 1,00 & & & 23,1 & 1,00 & & \\
\hline Test de tendencia $(\mathrm{p})$ & & 0,0001 & & & & 0,0001 & & \\
\hline Índice relativo de desigualdad & & 0,68 & $(0,61$ & $0,77)$ & & 0,56 & $(0,47$ & $0,66)$ \\
\hline \multicolumn{9}{|l|}{ Consulta al ginecólogo (2) } \\
\hline V. Bajo & 25,3 & 0,66 & $(0,56$ & $0,78)$ & 28,5 & 0,61 & $(0,52$ & $0,73)$ \\
\hline IV. Medio-Bajo & 39,6 & 0,70 & $(0,62$ & $0,80)$ & 43,1 & 0,70 & $(0,61$ & $0,80)$ \\
\hline III. Medio & 49,7 & 0,92 & $(0,83$ & $1,03)$ & 48,3 & 0,77 & $(0,68$ & $0,86)$ \\
\hline II. Medio-Alto & 52,4 & 0,92 & $(0,83$ & $1,02)$ & 50,3 & 0,80 & $(0,71$ & $0,90)$ \\
\hline I. Alto & 59,1 & 1,00 & & & 63,1 & 1,00 & & \\
\hline Test de tendencia $(p)$ & & 0,0001 & & & & 0,0001 & & \\
\hline Índice relativo de desigualdad & & 0,67 & $(0,61$ & $0,74)$ & & 0,66 & $(0,59$ & $0,74)$ \\
\hline
\end{tabular}

(1) La razón de porcentajes está ajustada por edad, sexo y presencia de alguna enfermedad 1987 no se obtuvo este tipo de información en la Encuesta Nacional de Salud. crónica en la consulta médica y en la hospitalización; por edad, sexo y estado de la dentadura en la consulta al dentista y por edad en la consulta al ginecólogo. RP: razón de porcentajes; IC 95\%:intervalo de confianza al 95\%.

(2) En la consulta al ginecólogo los datos no corresponden a 1987 sino a 1993, debido a que en 1987 no se obtuvo este tipo de información en la Encuesta Nacional de Salud.

dios pasó de 1,40 (intervalo de confianza IC: 1,22-1,62) en 1987 a 1,22 (IC: 1,03 $-1,43$ ) en $1995 / 97$ (tabla 2); por su parte, el índice relativo de desigualdad según el gru- po socioeconómico disminuyó de 1,44 (IC: $1,31-1,58)$ en el primer período a 1,18 (IC.1,05-1,32) en el segundo período (tabla 3$)$. 
Por otro lado, ni en 1987 ni en 1995/1997 se encontraron diferencias en la razón de porcentajes de hospitalización ni en el índice relativo de desigualdad. En cuanto a las consultas al dentista y al ginecólogo, la razón de porcentajes en ambos tipos de consulta disminuyó conforme se desciende en la categoría de nivel de estudios y de grupo socioeconómico y tanto su magnitud como su tendencia fueron estadísticamente significativas en ambos períodos $(p<0,05)$. En la consulta al dentista, el índice relativo de desigualdad pasó de 0,45 (IC: 0,39-0,52) a 0,53 (IC: $0,44-0,63$ ) en el caso del nivel de estudios (tabla 2) y de 0,68 (IC: $0,61-0,77)$ a 0,56 (IC:0,46-0,66) en el caso de grupo socioeconómico (tabla 3). Por su parte, en la consulta al ginecólogo, el índice relativo de desigualdad pasó de 0,70 (IC: $0,63-0,78)$ a $0,61($ IC:0,53-0,69) en el caso del nivel de estudios (tabla 2) y de 0,67 (IC: $0,61-0,74$ ) a 0,66 (IC:0,59-0,74) en el caso del grupo socioeconómico (tabla 3).
En las tablas 4 y 5 puede observarse el número de individuos que contestó a los tiempos de espera según el nivel de estudios y según el grupo socioeconómico, así como la media y mediana de los mismos. Por su parte, en la tabla 6 se muestra el decremento porcentual en los tiempos de espera con el incremento del nivel socioeconómico. En 1987, el tiempo de espera en la consulta y en el tiempo de espera para ingreso hospitalario ordinario presentaron el decremento porcentual de mayor magnitud: $-27 \%$ y $-30,8 \%$ en el caso del nivel de estudios y $-21,15$ y $-28,7 \%$ en el caso del grupo socioeconómico. Además este decremento fue estadísticamente significativo, si bien, en el análisis del tiempo de espera para ingreso hospitalario con el grupo socioeconómico, el valor de la $\mathrm{p}$ del coeficiente de regresión fue 0.0526. En 1995/97, únicamente el tiempo de espera en la consulta presentó un decremento porcentual asociado al aumento del nivel socioeconómico estadísticamente significativo: $-16,8 \%$ en el caso del nivel de estudios y $-12,3 \%$ en el caso del grupo socioeconómico.

Tabla 4

Número de individuos incluidos en el análisis según el nivel de estudios, media y percentil 50 del tiempo invertido en llegar a la consulta, del tiempo de espera en la consulta y del tiempo de espera para ingreso hospitalario ordinario Población española mayor de 24 años, 1987 y 1995/97

\begin{tabular}{|c|c|c|c|c|c|c|}
\hline & \multicolumn{3}{|c|}{1987} & \multicolumn{3}{|c|}{ 1995/97 } \\
\hline & $\begin{array}{l}\text { Número de } \\
\text { individuos }\end{array}$ & Media & Percentil 50 & $\begin{array}{l}\text { Número de } \\
\text { individuos }\end{array}$ & Media & Percentil 50 \\
\hline \multicolumn{7}{|c|}{$\begin{array}{l}\text { Tiempo de llegada a la consulta } \\
\text { (minutos) }\end{array}$} \\
\hline Sin estudios/primer grado & 5.510 & 18,3 & 10,0 & 1.930 & 15,8 & 10,0 \\
\hline Segundo grado/tercer grado & 2.496 & 17,3 & 10,0 & 846 & 14,6 & 10,0 \\
\hline \multicolumn{7}{|c|}{$\begin{array}{l}\text { Tiempo de espera en la consulta } \\
\text { (minutos) }\end{array}$} \\
\hline Sin estudios/Primer grado & 4.821 & 33,5 & 30,0 & 1.757 & 24,6 & 15,0 \\
\hline Segundo grado/Tercer grado & 2.199 & 27,4 & 20,0 & 763 & 22,3 & 15,0 \\
\hline \multicolumn{7}{|c|}{$\begin{array}{l}\text { Tiempo de espera para ingreso } \\
\text { hospitalario (1) }\end{array}$} \\
\hline Sin estudios/Primer grado & 480 & 51,5 & 15,0 & 181 & 7,9 & 4,0 \\
\hline Segundo grado/Tercer grado & 215 & 50,2 & 8,0 & 47 & 6,1 & 3,0 \\
\hline
\end{tabular}

(1) En 1987 se midió en días y en 1995/97 en meses. 
Tabla 5

Número de individuos incluidos en el análisis según el grupo socioeconómico, media y percentil 50 del tiempo invertido en llegar a la consulta, del tiempo de espera en la consulta y del tiempo de espera para ingreso hospitalario ordinario Población española mayor de 24 años, 1987 y 1995/97

\begin{tabular}{|c|c|c|c|c|c|c|}
\hline & \multicolumn{3}{|c|}{1987} & \multicolumn{3}{|c|}{ 1995/97 } \\
\hline & $\begin{array}{l}\text { Número de } \\
\text { individuos }\end{array}$ & Media & Percentil 50 & $\begin{array}{l}\text { Número de } \\
\text { individuos }\end{array}$ & Media & Percentil 50 \\
\hline \multicolumn{7}{|c|}{$\begin{array}{l}\text { Tiempo de llegada a la consulta } \\
\text { (minutos) }\end{array}$} \\
\hline V. Bajo/IV.Medio-bajo & 3.116 & 18,6 & 15,0 & 1.187 & 16,0 & 10,0 \\
\hline III. Medio & 2.239 & 17,5 & 10,0 & 844 & 15,0 & 10,0 \\
\hline II. Medio-Alto/I. Alto & 1.997 & 17,7 & 10,0 & 861 & 15,6 & 10,0 \\
\hline \multicolumn{7}{|c|}{$\begin{array}{l}\text { Tiempo de espera en la consulta } \\
\text { (minutos) }\end{array}$} \\
\hline V. Bajo/IV. Medio-bajo & 2.713 & 34,4 & 30,0 & 1.068 & 25,2 & 20,0 \\
\hline III. Medio & 1.944 & 31,0 & 30,0 & 768 & 24,8 & 15,0 \\
\hline II. Medio-Alto/I. Alto & 1.800 & 28,2 & 20,0 & 786 & 22,0 & 15,0 \\
\hline \multicolumn{7}{|c|}{$\begin{array}{l}\text { Tiempo de espera para ingreso } \\
\text { hospitalario (1) }\end{array}$} \\
\hline V. Bajo/IV. Medio-bajo & 280 & 49,2 & 15,0 & 106 & 8,6 & 4,0 \\
\hline III. Medio & 195 & 63,1 & 15,0 & 81 & 7,2 & 4,0 \\
\hline II. Medio-Alto/I. Alto & 180 & 39,0 & 11,0 & 45 & 6,5 & 3,0 \\
\hline
\end{tabular}

(1) En 1987 se midió en días y en 1995/97 en meses.

Tabla 6

Decremento porcentual en los tiempos de espera por cada unidad de aumento en el nivel socioeconómico Población española mayor de 24 años, 1987 y 1995/97

\begin{tabular}{|ccccccccccc}
\hline & $\begin{array}{c}\text { Decremento } \\
\text { porcentual (1) }\end{array}$ & IC 95\% & $p$ & $\begin{array}{c}\text { Decremento } \\
\text { porcentual (1) }\end{array}$ & IC 95\% & $p$ \\
\hline Nivel de estudios (2 categorías) & & & & & & & & & \\
Tiempo en llegar a la consulta & $-3,7$ & $(-9,4$ & $2,3)$ & 0,2237 & $-2,3$ & $(-10,4$ & $6,4)$ & 0,5866 \\
Tiempo de espera en la consulta & $-27,0$ & $(-32,4$ & $-21,0)$ & 0,0001 & $-16,8$ & $(-24,7$ & $-8,0)$ & 0,0003 \\
Tiempo de espera para ingreso & $-30,8$ & $(-51,9$ & $-0,4)$ & 0,0478 & $-19,1$ & $(-47,7$ & $25,2)$ & 0,3410 \\
\hline Grupo socioeconómico (3 categorías) & & & & & & & & \\
Tiempo en llegar a la consulta & $-9,3$ & $(-14,1$ & $-4,2)$ & 0,0005 & 1,8 & $(-5,7$ & $9,8)$ & 0,6519 \\
Tiempo de espera en la consulta & $-21,1$ & $(-26,4$ & $-15,3)$ & 0,0001 & $-12,3$ & $(-19,8$ & $-4,1)$ & 0,0039 \\
Tiempo de espera para ingreso & $-28,7$ & $(-49,3$ & $0,3)$ & 0,0526 & $-12,1$ & $(-39,1$ & $26,7)$ & 0,4956 \\
\hline
\end{tabular}

(1) Decremento porcentual ajustado por edad, sexo y presencia de enfermedad crónica. 


\section{DISCUSIÓN}

La frecuencia de consulta médica en España en la segunda mitad de los años noventa presenta el mismo patrón que en la segunda mitad de los años ochenta ${ }^{18,19}$ : los grupos de nivel socioeconómico más bajos tienen mayor tasa de consulta al médico que los grupos socioeconómicos más altos a igual grado de necesidad. Se mantiene, por tanto, una mayor utilización por parte de los grupos socioeconómicos bajos, si bien la magnitud de la relación disminuyó en 1995/97 con respecto a 1987. Una posible explicación de esta reducción podría ser al aumento en las personas con cobertura sanitaria doble (pública y privada) que ha ocurrido entre el primer y el segundo período analizado $^{22}$; además, el porcentaje de personas con doble cobertura es más alta en los individuos con mayor nivel de estudios y/o con mayor nivel de ingresos ${ }^{23}$. En cualquier caso, esto no descarta otras posibles explicaciones que pudieran obtenerse de otras investigaciones dirigidas a valorar de manera detallada esa tendencia.

Estudios realizados en otros países han mostrado que el principal número de visitas para el cuidado de la salud son al médico general y que las personas pertenecientes a niveles socioeconómicos más bajos presentan una mayor utilización de tales servi $\operatorname{cios}^{4,7,24-26}$. Una posible explicación de este hecho que podría plantearse es que las personas pertenecientes a los grupos socioeconómicos bajos serían más proclives a descargar la responsabilidad de sus problemas de salud y otras necesidades sociales en el sistema sanitario. Asimismo, cuestionarían en menor grado la autoridad y juicios de los médicos, asumiendo en gran medida que los profesionales sanitarios aliviarán sus síntomas y les curarán. Por el contrario, los grupos socioeconómicos más altos serían más discriminativos en la utilización de la consulta al médico y preferirían mantener cierto grado de control sobre su propia salud. En este sentido, asumirían más responsabilidad acerca de su propia salud y, por tanto, se sentirían menos inclinados a ir a la consulta del médico. Igualmente, las creencias pueden tener un impacto en la utilización de los servicios sanitarios independientemente de las medidas financieras tomadas y de cómo sea la estructura de la organización de los servicios de salud ${ }^{27}$. Como han sugerido algunos autores, las personas de grupos socioeconómicos más altos y de mayor nivel de estudios pueden articular mejor sus propias necesidades de salud ó sus demandas ó necesidades de atención sanitaria y recibir tratamientos más adecuados y efectivos que los del resto de los grupos socioeconómi$\cos ^{28}$.

En cuanto a la hospitalización, los resultados son similares en los dos períodos analizados: no se observan diferencias en la frecuencia de hospitalización según las características socioeconómicas de la población. Estudios realizados en otros países con cobertura universal de la asistencia sanitaria tampoco han encontrado diferencias socioeconómicas en la utilización de estos servi$\operatorname{cios}^{4,6,7,25,26,29}$. Posiblemente, en los sistemas sanitarios universales, la hospitalización, al contrario que la consulta al médico general, dependa de la gravedad de los problemas de salud y quizá, por esa razón, no se encuentren diferencias socioeconómicas en el uso de este servicio de salud.

Con respecto a la consulta al dentista la frecuencia de visitas fue menor en los grupos de nivel socioeconómico más bajos, tanto en 1987 como en 1995/97. Estudios realizados en España ${ }^{11,30}$ y en otros países han confirmado también de forma clara una mayor frecuencia de utilización de este tipo de servicio por parte de personas con mayores niveles de educación y de grupos socioeconómicos más altos $4,9,12,18,19,25,31$. Como han señalado otros autores, debido a que se trata de un servicio no cubierto por medio de financiación pública, sería la capacidad de pago por parte de las personas lo que determinaría y establecería la demanda de este tipo de servicios. En este sentido, tanto el nivel de estudios como los grupos socioeco- 
nómicos utilizados mostraron una fuerte relación con los ingresos del hogar, tanto en el primer período como en el segundo ${ }^{32}$.

Por lo que se refiere a la consulta al ginecólogo, se observó una mayor utilización por parte de las mujeres de niveles socioeconómicos altos, tanto en el primer período como en el segundo. Estas diferencias también se han observado en otros países con cobertura universal de la asistencia sanitaria $^{5,9,10,29} \mathrm{y}$, probablemente, puedan ser debidas a consultas de tipo preventivo relacionadas con el cribado de cáncer de mama $^{33}$ y de cérvix uterino ${ }^{34}$. Distintos estudios han demostrado que los servicios de salud preventivos son utilizados en mayor medida por personas de nivel socioeconómico alto ${ }^{35,36}$. La existencia de barreras que limitan el acceso a la información sobre salud, lo que conlleva necesariamente una menor percepción de la necesidad de asistencia sanitaria preventiva $^{37}$, es la razón que podría explicar la menor utilización de estos servicios por parte de personas de nivel socioeconómico más bajo o con menor nivel de estudios. Además, se ha señalado que los comportamientos preventivos también van a depender en gran medida de la percepción que las personas tienen de la amenaza que dicha enfermedad constituye para ellas y de la eficacia que creen que el llevar a cabo esa conducta tendrá para reducir la amenaza ${ }^{38}$. Esas creencias dependen a su vez de los sistemas socio-culturales de pertenencia y de referen$\operatorname{cia}^{38}$. En España, a lo largo de los noventa, se han ido implantando de forma paulatina los programas de despistaje precoz de cáncer de mama en las comunidades autónomas $^{34}$; sin embargo, es improbable que en el segundo de los períodos estudiados se haya notado su impacto, debido a que la mayoría comenzó en la segunda mitad de los años noventa.

En cuanto a la accesibilidad a los servicios sanitarios hay que señalar que continúa la ausencia de diferencias en el tiempo de llegada a la consulta atribuibles a las características socioeconómicas. Asimismo se ha observado que persisten las diferencias en el tiempo de espera para la consulta. Posiblemente, los programas de cita previa que se han implantado en algunos servicios de salud que gestionan la asistencia sanitaria pública han tenido impacto en la disminución del tiempo de espera en la consulta, pero no han disminuido las diferencias de este tiempo de espera en los diferentes estratos socioeconómicos de población. Como explicación de este hecho se podría señalar que los individuos con mayor nivel socioeconómico y/o mayor nivel de estudios conocen mejor el funcionamiento del sistema sanitario, tienen una mayor posibilidad de resolver los problemas administrativos para acceder a los mismos y un mejor conocimiento y comunicación con el personal médico responsable en última instancia de la provisión de los servicios ${ }^{39-40}$. No obstante, es posible que estas ventajas no se manifiesten totalmente en los casos en los que la prestación de los servicios sanitarios no dependen exclusivamente de la demanda, sino que se realizan de manera programada, como es el caso de la gestión de las listas de espera para ingreso hospitalario ordinario. En este sentido, las medidas que se han puesto en marcha para disminuir las listas de espera quirúrgicas quizás han podido influir en la desaparición de las diferencias en el tiempo de espera para ingreso hospitalario ordinario en el segundo período analizado, según las características socioeconómicas de la población estudiada.

En la interpretación de los resultados hay que tener en cuenta algunas consideraciones en relación con el período de recogida de datos en las encuestas. Las entrevistas en las encuestas de 1987 y 1993 se realizaron en el mes de julio y febrero, respectivamente, mientras que las entrevistas de 1995 y 1997 se realizaron a lo largo de todo el año. Esto plantearía la posibilidad de un sesgo en la estimación de las diferencias socioeconómicas en la utilización de servicios sanitarios. No obstante, un estudio ${ }^{19}$ donde se compararon las diferencias socioeconómicas en la utilización de servicios sanitarios en 1987 y 
1993, con la información procedente de las encuestas nacionales de salud de esos años, comprobó que tal sesgo no existía a pesar de que las encuestas se realizaron en períodos del año diferentes.

Igualmente, en la interpretación de los resultados hay que considerar el método utilizado para asignar a los individuos a distintos grupos socioeconómicos. Se utilizó este indicador compuesto como aproximación a la capacidad económica de los individuos; de hecho, el coeficiente de correlación de Spearman entre esta variable y los ingresos mensuales familiares en la encuesta de 1997 fue de 0,62. Por otro lado, como el nivel de estudios, esta variable presenta la ventaja de un grado alto de exhaustividad, ya que permite incluir a la mayoría de los individuos en el análisis, frente a la clase social basada en la ocupación que sólo permite clasificar a las personas que tienen o han tenido un empleo. Concretamente, sólo un $8 \%$ de los entrevistados del primer período y un $2 \%$ de los entrevistados del segundo período fueron excluidos porque no fue posible asignarlos a ninguno de estos grupos.

Como conclusión, cuando se compara la utilización y accesibilidad de los servicios sanitarios en la segunda mitad de los años noventa con la segunda mitad de los años ochenta en España, se observa que las personas de los estratos socioeconómicos bajos o de menor nivel de estudios continúan presentando una mayor frecuencia de consultas médicas que el resto de los grupos para igual grado de necesidad, pero una menor frecuencia de consultas ginecológicas, posiblemente preventivas, y una menor frecuencia de consultas al dentista; por otro lado, ni en uno ni en otro período se han encontrado diferencias en la frecuencia de hospitalización. Persisten las diferencias en los tiempos de espera para la consulta, según características socioeconómicas, y han desaparecido las diferencias en el tiempo de espera para ingreso hospitalario ordinario.

\section{BIBLIOGRAFÍA}

1. Graham S. Socio-economic status, illness, and the use of medical services. Milbank Mem Fund Q 1957; 35:58-66.

2. Townsend P. Inequality and the health service. Lancet 1974; 868:1179-1189.

3. Aday LA and Andersen RM. The national profile of access to medical care: where do we stand? Am J Public Health 1984; 74:1331-1339.

4. Piperno A, and Di Orio F. Social differences in health and utilization of health services in Italy. Soc Sci Med 1990; 31:305-312.

5. Blaxter M. Equity and consultation rates in general practice. BMJ 1984; 288:1963-1967.

6. Manga P, Broyles RW, Angus DE. The determinants of hospital utilization under a universal public insurance program in Canada. Med Care 1987; 25:658-670.

7. Haynes R. Inequalities in health and health service use: evidence from the General Household Survey. Soc Sci Med 1991; 33:361-368.

8. Casanova C, Starfield B. Hospitalization of children and access to primary care: a cross-national comparison. Int J Health Serv 1995; 25:283-294.

9. La Vecchia C, Negri E, Pagano R, Decarli A. Education, prevalence of disease, and frequency of health care utilization J Epidemiol Community Health 1987; 41:161-165.

10. Katz SJ, Hofer TP. Socioeconomic disparities in preventive care persist despite universal coverage. JAMA 1994; 272:530-534.

11. Regidor E, De Mateo S, Gutiérrez-Fisac JL, Fernández de la Hoz K, Rodríguez C. Diferencias socioeconómicas en la utilización y accesibilidad de los servicios sanitarios en España. Med Clin (Barc) 1996; 107:285-288.

12. Mormiche P. L' accès aux soins: évolution des inégalités entre 1980 et 1991 . Économie et Statistique $1995 ; 282: 3-18$

13. Whitehead M, Evandrou M, Haglund B, Diderichsen F. As the health divide widens in Sweden and Britain, what's happening to access to care? BMJ 1997; 315:1006-1009.

14. Diderichsen F. Market reforms in health care and the sustainability of the welfare state. Health Policy 1995; 32:141-153.

Rev Esp Salud Pública 2001, Vol. 75, N. ${ }^{\circ} 2$ 
15. Whitehead M, Gustaffson R, Diderichsen F. Why is Sweden rethinking its NHS-style reforms? BMJ 1997; 315: 000-0.

16. Alonso J, Antó JM. Desigualdades en salud en Barcelona. Gac Sanit 1988; 2:4-12.

17. Borrell C. Evolució de les desigualtats socials en salut a la ciutat de Barcelona. [Tesis doctoral] Barcelona: Universitat Autònoma de Barcelona; 1995.

18. Fernández de la Hoz K, Leon D. Self-perceived health status and inequalities in use of health services in Spain. Int J Epidemiol 1996; 25:593-693.

19. Navarro V, Benach J. Comisión de Estudios de las Desigualdades Sociales en Salud en España. Desigualdades sociales en salud en España. Madrid: Ministerio de Sanidad y Consumo; 1996.

20. Urbanos RM. Evolución de la desigualdad de acceso y utilización en la Sanidad Pública española (1987-1993): una aproximación a la equidad a partir de las encuestas de salud [citado el 8 de marzo de 2001]. URL: http://www.ucm.es/BUCM/ cee/doc/9913/9913.htm.

21. SAS Institute Inc. SAS/STAT. User's Guide, Version $6,4^{\text {th }}$ edn., Vol. 2, SAS Institute Inc, Cary, Nc; 1989.

22. Regidor E, Gutiérrez-Fisac JL. Indicadores de Salud. Cuarta evaluación en España del programa regional europeo Salud para todos. Madrid: Ministerio de Sanidad y Consumo; 1999.p.287-288.

23. Ministerio de Sanidad y Consumo. Encuesta Nacional de Salud de España 1997. Madrid: Ministerio de Sanidad y Consumo; 1999.p.226-228.

24. Townsend P, Davidson N. Inequalities in Health: The Black Report. Harmondsworth: Penguin Books; 1982.

25. Keskimäki I, Salinto M, Aro S. Socioeconomic Equity in Finnish hospital care in relation to need. Soc Sci Med 1995; 41:425-431.

26. Van der Meer JBW, Van den Bos J, Mackenbach JP. Socioeconomic differences in the utilization of health services in a Dutch population: the contribution of health status. Health Policy 1996; 37:1-18.

27. Susser MW, Watson W. Sociology in Medicine. London: Oxford University Press; 1971.
28. Hexel PC, Wintersberger H. Inequalities in health: strategies. Soc Sci Med 1986; 22:151-160.

29. Mackenbach JP. Socio-economic health differences in the Netherlands: a review of recent empirical findings. Soc Sci Med 1992; 34:213-226.

30. Fernández-Mayoralas G, Rodríguez V, Rojo F. Health services accessibility among Spanish elderly. Soc Sci Med 2000; 50:17-26.

31. Grytten J, Holst D, Laake P. Accessibility of dental services according to family income in a noninsured population. Soc Sci Med 1993; 37:1501-1508.

32. Ayala L, Ruiz-Huerta J, Martínez R. El mercado de trabajo y la distribución personal de la renta en España en los años noventa. Ekonomiaz 1998; 40:105-133.

33. Lostao L. Health inequalities and participation in a breast cancer screening program. $7 .^{\circ}$ European Congress of the European Society of Health and Medical Sociology. Rennes, August 1998, p.259.

34. Ascunce N, Del Moral A. Detección precoz del cáncer de mama y del cáncer de cuello de útero. En: Navarro C, Cabasés JM, Tormo MJ. La salud y el sistema sanitario en España: Informe SESPAS 1995. Barcelona: SG Editore; 1995.p. 46-55.

35. Dutton DB. Explaining the low use of health services by the poor. Cost, attitudes, or delivery sistems? American Sociological Review 1978; $43: 348-368$

36. Dutton DB. Patterns of ambulatory health care in five different delivery systems. Med Care 1979; $17: 221-241$

37. Whitehead M. Health services: fair and equitable? En: Townsend P, Whitehead M, Davidson $\mathrm{N}$, editores. Inequalities in health. Londres: Penguin Books;1992.p. 277-286.

38. Lostao L. Factores predictores de la participación en un programa de «screening» de cáncer de mama: implicación del modelo de creencias de salud y de las variables sociodemográficas. Rev Esp Invest Sociol 2000; 92:173-192.

39. Adler NE, Boyce WT, Chesney AM, Folkman S, Syme L. Socioeconomic inequalities in health. JAMA 1993; 269:3140-3145.

40. Feinstein JS. The relationship between socioeconomic status and health: a review of the literature. Milbank Mem Fund Q 1993; 71:279-320. 